\title{
THE CARTAN, CHOQUET AND KELLOGG PROPERTIES FOR THE FINE TOPOLOGY ON METRIC SPACES
}

\author{
By \\ ANDERS BJÖRn, JANA BJÖRn, AND VISA LATVALA
}

\begin{abstract}
We prove the Cartan and Choquet properties for the fine topology on a complete metric space equipped with a doubling measure supporting a $p$ Poincaré inequality, $1<p<\infty$. We apply these key tools to establish a fine version of the Kellogg property, characterize finely continuous functions by means of quasicontinuous functions, and show that capacitary measures associated with Cheeger supersolutions are supported by the fine boundary of the set.
\end{abstract}

\section{Introduction}

The aim of this paper is to establish the Cartan and Choquet properties for the fine topology on a complete metric space $X$ equipped with a doubling measure $\mu$ supporting a $p$-Poincaré inequality, $1<p<\infty$. These properties are crucial for deep applications of the fine topology in potential theory. As applications of these key tools, we establish the fine Kellogg property and characterize finely continuous functions by means of quasicontinuous functions. We also show that capacitary measures associated with Cheeger $p$-supersolutions are supported by the fine boundary of the set (not just by the metric boundary).

The classical fine topology is closely related to the Dirichlet problem for the Laplace equation. Wiener [52] showed in 1924 that a boundary point of a domain is irregular if and only if the complement is thin at that point in a certain capacity density sense; cf. Definition 6.1. In 1939, Brelot [21], [22] characterized thinness by a condition which is nowadays called the Cartan property. The reason for this name is that Cartan (in a letter to Brelot in 1940, see [23, p. 14]) connected the notion of thinness to the coarsest topology making all superharmonic functions continuous. Cartan [25] coined the name fine topology for such a topology.

(C) The author(s) 2018. This article is published with open access at Springerlink.com

${ }^{1}$ The first two authors were supported by the Swedish Research Council. 
Nonlinear potential theory associated with $p$-harmonic functions has been studied since the 1960s. For extensive treatises and notes on the history, see the monographs [1], [36] and [48]. Starting in the 1990s a lot of attention has been given to analysis on metric spaces, see, e.g., [31], [34] and [37]. Around 2000, this initiated studies of $p$-harmonic and $p$-superharmonic functions on metric spaces without a differentiable structure; see, e.g., [9], [14], [42], [43] and [51].

The classical linear fine potential theory and fine topology (the case $p=2$ ) have been systematically studied since the 1960s. Let us here just mention [24], [29], [30] and[47], which include most of the theory and the main references. Some of these works are written in large generality including topological spaces, general capacities and families of functions, and some results thus apply also to the nonlinear theory. At the same time, many other results rely indirectly on a linear structure, e.g., through potentials, integral representations and convex cones of superharmonic functions, which are in general not available in the nonlinear setting.

The nonlinear fine potential theory started in the 1970 s on unweighted $\mathbf{R}^{n}$; see [36, notes to Chapter 12] and [48, Section 2.6]. For the fine potential theory associated with $p$-harmonic functions on unweighted $\mathbf{R}^{n}$, see [48] and [46]. The monograph [36] is the main source for fine potential theory on weighted $\mathbf{R}^{n}$ (note that Chapter 21, which is only in the second edition, contains some more recent results). The study of fine potential theory on metric spaces is more recent; see, e.g. [10], [11], [19], [41] and [44]. For further references to nonlinear and fine nonlinear potential theory, see the introduction to [11].

Recently, in [11], we established the so-called weak Cartan property, which says that if $E \subset X$ is thin at $x_{0} \notin E$, then there exist a ball $B \ni x_{0}$ and superharmonic functions $u, u^{\prime}$ on $B$ such that $v\left(x_{0}\right)<\liminf _{E \ni x \rightarrow x_{0}} v(x)$, where $v=$ $\max \left\{u, u^{\prime}\right\}$.

The superharmonic functions considered in [11] are based on upper gradients, and because of the lack of a differential equation, we did not succeed in obtaining the full Cartan property as in $\mathbf{R}^{n}$, where $v$ itself can be chosen superharmonic, cf. Theorem 1.1 below. Indeed, the proof of the full Cartan property seems to be as hard as the proof of the Wiener criterion, which is also open in the nonlinear potential theory based on upper gradients, but is known to hold in the potential theory based on Cheeger gradients; see [18]. Nevertheless, the weak Cartan property in [11] was enough to enable us to conclude that the fine topology is the coarsest one making all superharmonic functions continuous.

Here, we instead focus on Cheeger superharmonic functions based on Cheeger's theorem yielding a vector-valued Cheeger gradient. In this case, we 
do have an equation available, and this enables us to establish the following full Cartan property.

Theorem 1.1 (Cartan property). Suppose that $E$ is thin at $x_{0} \in \bar{E} \backslash E$. Then there is a bounded positive Cheeger superharmonic function $u$ in an open neighbourhood of $x_{0}$ such that

$$
u\left(x_{0}\right)<\liminf _{E \ni x \rightarrow x_{0}} u(x)
$$

For a Newtonian function, the minimal $p$-weak upper gradient and the modulus of the Cheeger gradient are comparable. Thus the corresponding capacities are comparable to each other, and the fine topology, as well as thinness (and thickness), is the same in both cases. Superminimizers, superharmonic and $p$ harmonic functions are, however, different. Hence, using the Cheeger structure, we can study thinness and the fine topology, but not e.g. the superharmonic and $p$-harmonic functions based on upper gradients. Only Cheeger $p$-(super)harmonic functions can be treated.

We use the Cartan property to establish the following important Choquet property.

Theorem 1.2 (Choquet property). For any $E \subset X$ and $\varepsilon>0$, there is an open set $G$ containing all the points in $X$ at which $E$ is thin such that $C_{p}(E \cap G)<\varepsilon$.

The Choquet property was first established by Choquet [27] in 1959. In the nonlinear theory on unweighted $\mathbf{R}^{n}$, it was later established by Hedberg [32] and Hedberg-Wolff [33] in connections with potentials (also for higher-order Sobolev spaces). The Cartan property for $p$-superharmonic functions on unweighted $\mathbf{R}^{n}$ was obtained by Kilpeläinen-Malý [40] as a consequence of their pointwise Wolffpotential estimates. In fact, Kilpeläinen and Malý used the Cartan property to establish the necessity in the Wiener criterion. In[48], Malý and Ziemer deduced the Choquet property from the Cartan property. The proof of the Cartan property was extended to weighted $\mathbf{R}^{n}$ by Mikkonen [49, Theorem 5.8] and can also be found in [36, Theorem 21.26 (which is only in the second edition)]; in both places, however, they refrained from deducing consequences such as the Choquet property.

Our proof of the Choquet property follows the one in [48], but we have some extra complications due to the possible presence of some points with zero capacity and others with positive capacity. Note that [29] contains a proof of the Choquet property in an axiomatic setting, assuming Corollary 1.3 and Theorem 1.4(a). We have a converse approach, since our proofs of Corollary 1.3 and Theorem 1.4 are based on the Choquet property. 
Corollary 1.3 (Fine Kellogg property). For every $E \subset X$,

$$
C_{p}(\{x \in E: E \text { is thin at } x\})=0 \text {. }
$$

The fine Kellogg property has close connections with boundary regularity; see Remark 7.3. The "only if" implications in the following result were already obtained in [11], but now we are able to complete the picture.

Theorem 1.4. (a) A set $U \subset X$ is quasiopen if and only if $U=V \cup E$ for some finely open set $V$ and for a set $E$ of capacity zero.

(b) An extended real-valued function on a quasiopen set $U$ is quasicontinuous in $U$ if and only if $u$ is finite q.e. and finely continuous q.e. in $U$.

It is pointed out in [2, Proposition 3] that (a) for unweighted $\mathbf{R}^{n}$ follows from the Choquet property established in [33]. Also, (b) then follows by a modification of the earlier axiomatic arguments of Fuglede [29, Lemma, p. 143]. The proof of Theorem 1.4 in unweighted $\mathbf{R}^{n}$ is given in [48, p. 146]. For the reader's convenience, we include the proof of Theorem 1.4 although the proof essentially follows [48]. In Section 8, we use Theorem 1.4 to extend and simplify some recent results from [10].

We end the paper with another application of the Cartan property in Section 9, which contains results on capacitary measures related to Cheeger supersolutions; see Theorem 9.1 and Corollaries 9.5 and 9.6. In particular, we show that the capacitary measure only charges the fine boundary of the corresponding set. This seems to be new also in unweighted $\mathbf{R}^{n}$.

\section{Notation and preliminaries}

We assume hroughout the paper that $1<p<\infty$ and that $X=(X, d, \mu)$ is a metric space equipped with a metric $d$ and a positive complete Borel measure $\mu$ such that $0<\mu(B)<\infty$ for all (open) balls $B \subset X$. It follows that $X$ is separable. The $\sigma$-algebra on which $\mu$ is defined is obtained by the completion of the Borel $\sigma$-algebra. We also assume that $\Omega \subset X$ is a nonempty open set.

We say that $\mu$ is doubling if there exists a doubling constant $C>0$ such that for all balls $B=B\left(x_{0}, r\right):=\left\{x \in X: d\left(x, x_{0}\right)<r\right\}$,

$$
0<\mu(2 B) \leq C \mu(B)<\infty .
$$

Here and elsewhere, we let $\delta B=B\left(x_{0}, \delta r\right)$. A metric space with a doubling measure is proper (i.e., closed and bounded subsets are compact) if and only if it is complete. See [34] for more on doubling measures. 
A curve is a continuous mapping from an interval, and a rectifiable curve is a curve of finite length. We consider only curves which are nonconstant, compact and rectifiable. Such a curve can be parameterized by its arc length $d s$. We follow Heinonen and Koskela [37] in introducing upper gradients (they called them very weak gradients) as follows.

Definition 2.1. A nonnegative Borel function $g$ on $X$ is an upper gradient of an extended real-valued function $f$ on $X$ if for all nonconstant, compact and rectifiable curves $\gamma:\left[0, l_{\gamma}\right] \rightarrow X$,

$$
\left|f(\gamma(0))-f\left(\gamma\left(l_{\gamma}\right)\right)\right| \leq \int_{\gamma} g d s
$$

where we follow the convention that the left-hand side is $\infty$ whenever at least one of the terms therein is infinite.

If $g$ is a nonnegative measurable function on $X$ and if (2.1) holds for $p$-almost every curve (see below), then $g$ is a $p$-weak upper gradient of $f$.

Here we say that a property holds for $p$-almost every curve if it fails only for a curve family $\Gamma$ with zero $p$-modulus, i.e. there exists $0 \leq \rho \in L^{p}(X)$ such that $\int_{\gamma} \rho d s=\infty$ for every curve $\gamma \in \Gamma$. Note that a $p$-weak upper gradient need not be a Borel function; it is only required to be measurable. On the other hand, every measurable function $g$ can be modified on a set of measure zero to give a Borel function, from which it follows that $\int_{\gamma} g d s$ is defined (with a value in $[0, \infty]$ ) for $p$-almost every curve $\gamma$. For proofs of these and all other facts in this section, we refer to [9] and [38].

The $p$-weak upper gradients were introduced in [45]. It was also shown there that if $g \in L_{\mathrm{loc}}^{p}(X)$ is a $p$-weak upper gradient of $f$, then one can find a sequence $\left\{g_{j}\right\}_{j=1}^{\infty}$ of upper gradients of $f$ such that $g_{j}-g \rightarrow 0$ in $L^{p}(X)$. If $f$ has an upper gradient in $L_{\mathrm{loc}}^{p}(X)$, then it has a minimal $p$-weak upper gradient $g_{f} \in L_{\mathrm{loc}}^{p}(X)$ in the sense that for every $p$-weak upper gradient $g \in L_{\text {loc }}^{p}(X)$ of $f, g_{f} \leq g$ a.e.; see [51]. The minimal $p$-weak upper gradient is well-defined up to a set of measure zero in the cone of nonnegative functions in $L_{\mathrm{loc}}^{p}(X)$. Following [50], we define a version of Sobolev spaces on the metric measure space $X$.

Definition 2.2. For measurable $f$, let

$$
\|f\|_{N^{1, p}(X)}=\left(\int_{X}|f|^{p} d \mu+\inf _{g} \int_{X} g^{p} d \mu\right)^{1 / p},
$$

where the infimum is taken over all upper gradients of $f$. The Newtonian space on $X$ is

$$
N^{1, p}(X)=\left\{f:\|f\|_{N^{1, p}(X)}<\infty\right\} .
$$


The space $N^{1, p}(X) / \sim$, where $f \sim h$ if and only if $\|f-h\|_{N^{1, p}(X)}=0$, is a Banach space and a lattice; see [50]. In this paper, we assume that functions in $N^{1, p}(X)$ are defined everywhere (with values in $\overline{\mathbf{R}}:=[-\infty, \infty]$ ), not just up to an equivalence class in the corresponding function space. For a measurable set $E \subset X$, the Newtonian space $N^{1, p}(E)$ is defined by considering $\left(E,\left.d\right|_{E},\left.\mu\right|_{E}\right)$ as a metric space in its own right. We say that $f \in N_{\text {loc }}^{1, p}(E)$ if, for every $x \in E$, there exists a ball $B_{x} \ni x$ such that $f \in N^{1, p}\left(B_{x} \cap E\right)$. If $f, h \in N_{\mathrm{loc}}^{1, p}(X)$, then $g_{f}=g_{h}$ a.e. in $\{x \in X: f(x)=h(x)\}$; in particular, $g_{\min \{f, c\}}=g_{f} \chi_{\{f<c\}}$ for $c \in \mathbf{R}$.

Definition 2.3. The Sobolev capacity of an arbitrary set $E \subset X$ is

$$
C_{p}(E)=\inf _{u}\|u\|_{N^{1, p}(X)}^{p},
$$

where the infimum is taken over all $u \in N^{1, p}(X)$ such that $u \geq 1$ on $E$.

The Sobolev capacity is countably subadditive. We say that a property holds quasieverywhere (q.e.) if the set of points for which the property does not hold has Sobolev capacity zero. The Sobolev capacity is the correct gauge for distinguishing between two Newtonian functions. If $u \in N^{1, p}(X)$, then $u \sim v$ if and only if $u=v$ q.e. Moreover, [50, Corollary 3.3] shows that if $u, v \in N^{1, p}(X)$ and $u=v$ a.e., then $u=v$ q.e.

A set $U \subset X$ is quasiopen if for every $\varepsilon>0$ there is an open set $G \subset X$ such that $C_{p}(G)<\varepsilon$ and $G \cup U$ is open. A function $u$ defined on a set $E \subset X$ is quasicontinuous if for every $\varepsilon>0$ there is an open set $G \subset X$ such that $C_{p}(G)<\varepsilon$ and $\left.u\right|_{E \backslash G}$ is finite and continuous. It is easily verified that if $u$ is quasicontinuous on a quasiopen set $U$, then the sets $\{x \in U: u(x)<a\}$ and $\{x \in U: u(x)>a\}$ are quasiopen for all $a \in \mathbf{R}$.

Definition 2.4. We say that $X$ supports a $p$-Poincaré inequality if there exist constants $C>0$ and $\lambda \geq 1$ such that for all balls $B \subset X$, all integrable functions $f$ on $X$ and all upper gradients $g$ of $f$,

$$
f_{B}\left|f-f_{B}\right| d \mu \leq C \operatorname{diam}(B)\left(f_{\lambda B} g^{p} d \mu\right)^{1 / p},
$$

where $f_{B}:=f_{B} f d \mu:=\int_{B} f d \mu / \mu(B)$.

In the definition of Poincaré inequality, we can equivalently assume that $g$ is a $p$-weak upper gradient.

In $\mathbf{R}^{n}$ equipped with a doubling measure $d \mu=w d x$ ( $d x$ denotes Lebesgue measure), the $p$-Poincaré inequality (2.2) is equivalent to the $p$-admissibility of the weight $w$ in the sense of [36], cf. [36, Corollary 20.9] and [9, Proposition A.17]. 
If $X$ is complete and supports a $p$-Poincaré inequality and $\mu$ is doubling, then Lipschitz functions are dense in $N^{1, p}(X)$; see [50]. Moreover, all functions in $N^{1, p}(X)$ and those in $N^{1, p}(\Omega)$ are quasicontinuous; see [15]. This means that in the euclidean setting, $N^{1, p}\left(\mathbf{R}^{n}\right)$ is the refined Sobolev space as defined in [36, p. 96]; see [9, Appendix A.2] for a proof of this fact in weighted $\mathbf{R}^{n}$. This is the main reason that, unlike in the classical euclidean setting, we do not need to require the functions competing in the definitions of capacity to be 1 in a neighbourhood of $E$. For recent related progress on the density of Lipschitz functions, see [3] and [4].

In Section 6, the fine topology is defined by means of thin sets, which in turn use the variational capacity $\operatorname{cap}_{p}$. To be able to define the variational capacity, we first need a Newtonian space with zero boundary values. For an arbitrary set $A \subset X$, we let

$$
N_{0}^{1, p}(A)=\left\{\left.f\right|_{A}: f \in N^{1, p}(X) \text { and } f=0 \text { on } X \backslash A\right\} .
$$

One can replace the assumption " $f=0$ on $X \backslash A$ " with " $f=0$ q.e. on $X \backslash A$ " without changing the obtained space $N_{0}^{1, p}(A)$. Functions from $N_{0}^{1, p}(A)$ can be extended by zero in $X \backslash A$, and we regard them in that sense if needed.

Definition 2.5. The variational capacity of $E \subset \Omega$ with respect to $\Omega$ is

$$
\operatorname{cap}_{p}(E, \Omega)=\inf _{u} \int_{X} g_{u}^{p} d \mu
$$

where the infimum is taken over all $u \in N_{0}^{1, p}(\Omega)$ such that $u \geq 1$ on $E$.

If $C_{p}(E)=0$, then $\operatorname{cap}_{p}(E, \Omega)=0$. The converse implication is true if $\mu$ is doubling and supports a $p$-Poincaré inequality, $\Omega$ is bounded, and $C_{p}(X \backslash \Omega)>0$.

We need the following simple lemma in Section 9. For the reader's convenience, we provide the short proof.

Lemma 2.6. If $u, v \in N^{1, p}(X)$ are bounded, then $u v \in N^{1, p}(X)$.

Proof. We can assume that $|u|$ and $|v|$ are bounded by 1 . Then $|u v| \leq|u|$, and hence $u v \in L^{p}(X)$. By the Leibniz rule ([9, Theorem 2.15]), $g:=|u| g_{v}+|v| g_{u}$ is a $p$-weak upper gradient of $u v$. As $g \leq g_{v}+g_{u} \in L^{p}(X)$, we see that $u v \in N^{1, p}(X)$.

Throughout the paper, the letter $C$ denotes various positive constants whose value may vary even within a line. We also write $A \simeq B$ if $C^{-1} A \leq B \leq C A$. 


\section{Cheeger gradients}

Throughout the rest of the paper, we assume that $X$ is complete and supports a p-Poincaré inequality and that $\mu$ is doubling.

In addition to upper gradients, we also use Cheeger gradients. Their existence is based on the following deep result of Cheeger.

Theorem 3.1 ([26, Theorem 4.38]). There exist $N$ and a countable collection $\left(U_{\alpha}, X^{\alpha}\right)$ of pairwise disjoint measurable sets $U_{\alpha}$ and Lipschitz "coordinate" functions $X^{\alpha}: X \rightarrow \mathbf{R}^{k(\alpha)}, 1 \leq k(\alpha) \leq N$, such that $\mu\left(X \backslash \bigcup_{\alpha} U_{\alpha}\right)=0$ and for every Lipschitz function $f: X \rightarrow \mathbf{R}$ there exist unique bounded vector-valued functions $d^{\alpha} f: U_{\alpha} \rightarrow \mathbf{R}^{k(\alpha)}$ such that for a.e. $x \in U_{\alpha}$,

$$
\lim _{r \rightarrow 0} \sup _{y \in B(x, r)} \frac{\left|f(y)-f(x)-d^{\alpha} f(x) \cdot\left(X^{\alpha}(y)-X^{\alpha}(x)\right)\right|}{r}=0,
$$

where denotes the usual inner product in $\mathbf{R}^{k(\alpha)}$.

Cheeger (see [26, p. 460]) further shows that for a.e. $x \in U_{\alpha}$, there is an inner product norm $|\cdot|_{x}$ on $\mathbf{R}^{k(\alpha)}$ such that for all Lipschitz $f$,

$$
\frac{1}{C} g_{f}(x) \leq\left|d^{\alpha} f(x)\right|_{x} \leq C g_{f}(x),
$$

where $C$ is independent of $f$ and $x$. As Lipschitz functions are dense in $N^{1, p}(X)$, the "gradients" $d^{\alpha} f$ extend uniquely to the whole $N^{1, p}(X)$, by [28, Theorem 10] or [39]. Moreover, (3.1) holds even for functions in $N^{1, p}(X)$.

From now on, we drop $\alpha$ and set $D f:=d^{\alpha} f$ in $U_{\alpha}$.

There is some freedom in choosing the Cheeger structure on a metric space. However, on $\mathbf{R}^{n}$ we always make the natural choice $D f=\nabla f$ and let the inner product norm in (3.1) be the euclidean norm. Here, $\nabla f$ denotes the Sobolev gradient from [36], which equals the distributional gradient if the weight on $\mathbf{R}^{n}$ is a Muckenhoupt $A_{p}$ weight. In this case, $|D f|=g_{f}$, by [9, Proposition A.13].

\section{Supersolutions and superharmonic functions}

In the literature on potential theory on metric spaces, one usually studies the following (super)minimizers based on upper gradients.

Definition 4.1. A function $u \in N_{\mathrm{loc}}^{1, p}(\Omega)$ is a (super)minimizer on $\Omega$ if

$$
\int_{\{\varphi \neq 0\}} g_{u}^{p} d \mu \leq \int_{\{\varphi \neq 0\}} g_{u+\varphi}^{p} d \mu \quad \text { for all (nonnegative) } \varphi \in \operatorname{Lip}_{c}(\Omega) .
$$

A $p$-harmonic function is a continuous minimizer. 
Here $\operatorname{Lip}_{c}(\Omega)=\{\varphi \in \operatorname{Lip}(X): \operatorname{supp} \varphi \Subset \Omega\}$ and $E \Subset \Omega$ if $\bar{E}$ is a compact subset of $\Omega$.

Minimizers were first studied by Shanmugalingam [51], and superminimizers by Kinnunen-Martio [42]. For various characterizations of minimizers and superminimizers, see [6]. If $u$ is a superminimizer, then its lsc-regularization

$$
u^{*}(x):=\text { ess } \lim _{y \rightarrow x} \inf u(y)=\lim _{r \rightarrow 0} \underset{B(x, r)}{\operatorname{ess} \inf } u
$$

is also a superminimizer and $u^{*}=u$ q.e.; see [42] or [13]. If $u$ is a minimizer, then $u^{*}$ is continuous (by [43] or [16]), and thus $p$-harmonic. For further discussion and references on the topics in this section, see [9].

In this paper, we consider Cheeger (super)minimizers and Cheeger $p$ harmonic functions, defined by replacing $g_{u}$ and $g_{u+\varphi}$ in Definition 4.1 by $|D u|$ and $|D(u+\varphi)|$, respectively, where $|\cdot|$ is the inner product norm in (3.1). Due to the vector structure of the Cheeger gradient, one can also make the following definition. (There is no corresponding notion for upper gradients.)

Definition 4.2. A function $u \in N_{\text {loc }}^{1, p}(\Omega)$ is a (super)solution on $\Omega$ if

$$
\int_{\Omega}|D u|^{p-2} D u \cdot D \varphi d \mu \geq 0 \quad \text { for all (nonnegative) } \varphi \in \operatorname{Lip}_{c}(\Omega)
$$

where is the inner product giving rise to the norm in (3.1).

It can be shown that a function is a (super)solution if and only if it is a Cheeger (super)minimizer; the proof is the same as for [36, Theorem 5.13]. In weighted $\mathbf{R}^{n}$, with the choice $D f=\nabla f$, we have $g_{f}=|D f|=|\nabla f|$ a.e., which implies that (super)minimizers, Cheeger (super)minimizers and (super)solutions coincide and are the same as in [36].

We consider the following obstacle problem.

Definition 4.3. Let $\Omega \subset X$ be a nonempty bounded open set such that $C_{p}(X \backslash \Omega)>0$. For $f \in N^{1, p}(\Omega)$ and $\psi: \Omega \rightarrow \overline{\mathbf{R}}$, let

$$
\mathcal{K}_{\psi, f}(\Omega)=\left\{v \in N^{1, p}(\Omega): v-f \in N_{0}^{1, p}(\Omega) \text { and } v \geq \psi \text { q.e. in } \Omega\right\} .
$$

A function $u \in \mathcal{K}_{\psi, f}(\Omega)$ is a solution of the $\mathcal{K}_{\psi, f}(\Omega)$-Cheeger obstacle problem if

$$
\int_{\Omega}|D u|^{p} d \mu \leq \int_{\Omega}|D v|^{p} d \mu \quad \text { for all } v \in \mathcal{K}_{\psi, f}(\Omega) .
$$

If $\mathcal{K}_{\psi, f}(\Omega) \neq \varnothing$, then there is a solution $u$ of the $\mathcal{K}_{\psi, f}(\Omega)$-Cheeger obstacle problem, and this solution is unique up to equivalence in $N^{1, p}(\Omega)$. Moreover, $u^{*}$ is 
the unique lsc-regularized solution. Conditions for $\mathcal{K}_{\psi, f}(\Omega) \neq \varnothing$ can be found in [10]. If the obstacle $\psi$ is continuous, as a function with values in $[-\infty, \infty)$, then $u^{*}$ is also continuous. These results were obtained for the upper gradient obstacle problem by Kinnunen-Martio [42], where superharmonic functions based on upper gradients were also introduced. As with most of the results in the metric theory, their proofs work verbatim for the Cheeger case considered here. Since most of the theory has been developed in the setting of upper gradients, we often just refer to the upper gradient equivalents of results for Cheeger (super)minimizers.

Definition 4.4. The Cheeger capacitary potential $u_{E}$ of a set $E \subset \Omega$ in the bounded open set $\Omega$ with $C_{p}(X \backslash \Omega)>0$ is the lsc-regularized solution of the $\mathcal{K}_{\chi_{E}, 0}(\Omega)$-Cheeger obstacle problem. The Cheeger variational capacity of $E \subset \Omega$ is defined as

$$
\mathrm{Ch}_{-\operatorname{cap}_{p}}(E, \Omega)=\int_{X}\left|D u_{E}\right|^{p} d \mu=\inf _{u} \int_{X}|D u|^{p} d \mu,
$$

where the infimum is taken over all $u \in N_{0}^{1, p}(\Omega)$ such that $u \geq 1$ on $E$.

By (3.1), we have

$$
\mathrm{Ch}_{-\operatorname{cap}_{p}}(E, \Omega) \simeq \operatorname{cap}_{p}(E, \Omega) .
$$

For $f \in N^{1, p}(\Omega)$, we denote by $H_{\Omega} f$ the continuous solution of the $\mathcal{K}_{-\infty, f}(\Omega)$ Cheeger obstacle problem. This function is Cheeger $p$-harmonic in $\Omega$ and has the same boundary values (in the Sobolev sense) as $f$ on $\partial \Omega$, and hence is also called the solution of the (Cheeger) Dirichlet problem with Sobolev boundary values.

A solution of the $\mathcal{K}_{\psi, f}(\Omega)$-Cheeger obstacle problem is easily seen to be a Cheeger superminimizer (i.e. a supersolution) on $\Omega$. Conversely, a supersolution $u$ on any open $\Omega$ is a solution of the $\mathcal{K}_{u, u}\left(\Omega^{\prime}\right)$-Cheeger obstacle problem for all open $\Omega^{\prime} \Subset \Omega$ with $C_{p}\left(X \backslash \Omega^{\prime}\right)>0$.

Definition 4.5. Let $\Omega \subset X$ be an open set. A function $u: \Omega \rightarrow(-\infty, \infty]$ is Cheeger superharmonic in $\Omega$ if

(i) $u$ is lower semicontinuous;

(ii) $u$ is not identically $\infty$ in any component of $\Omega$;

(iii) for every nonempty open set $\Omega^{\prime} \Subset \Omega$ with $C_{p}\left(X \backslash \Omega^{\prime}\right)>0$ and all functions $v \in \operatorname{Lip}(X)$, we have $H_{\Omega^{\prime}} v \leq u$ in $\Omega^{\prime}$ whenever $v \leq u$ on $\partial \Omega^{\prime}$.

This definition of Cheeger superharmonicity is equivalent to the one in [36]; see [5]. A locally bounded Cheeger superharmonic function is a supersolution, and all Cheeger superharmonic functions are lsc-regularized. Conversely, any lscregularized supersolution is Cheeger superharmonic; see [42]. 


\section{Supersolutions and Radon measures}

In this section, we assume that $\Omega$ is a nonempty bounded open set and that $C_{p}(X \backslash \Omega)>0$.

A Radon measure is a positive complete Borel measure which is finite on every compact set. It was shown in [20, Propositions 3.5 and 3.9] that there is a one-to-one correspondence between supersolutions on $\Omega$ and Radon measures in the dual $N_{0}^{1, p}(\Omega)^{\prime}$.

Proposition 5.1. For every supersolution u on $\Omega$, there is a Radon measure $\nu \in N_{0}^{1, p}(\Omega)^{\prime}$ such that for all $\varphi \in N_{0}^{1, p}(\Omega)$,

$$
T u(\varphi):=\int_{\Omega}|D u|^{p-2} D u \cdot D \varphi d \mu=\int_{\Omega} \varphi d v,
$$

where is the inner product giving rise to the norm in (3.1). Conversely, if $v \in N_{0}^{1, p}(\Omega)^{\prime}$ is a Radon measure on $\Omega$, then there exists a unique lsc-regularized $u \in N_{0}^{1, p}(\Omega)$ satisfying $T u=v$ in the sense of (5.1) for all $\varphi \in N_{0}^{1, p}(\Omega)$. Moreover, $u$ is a nonnegative supersolution on $\Omega$.

Remark 5.2. The conclusion of Proposition 5.1 is false without the assumption $C_{p}(X \backslash \Omega)>0$. Indeed, if $u$ is a nonnegative lsc-regularized supersolution on $\Omega$, then $u$ is Cheeger superharmonic on $\Omega$. If $C_{p}(X \backslash \Omega)=0$, then $u$ has a Cheeger superharmonic extension to $X$, by [7, Theorem 6.3] (or [9, Theorem 12.3]), which must be constant, by [9, Corollary 9.14]. (Note that if $\Omega$ is bounded and $C_{p}(X \backslash \Omega)=0$, then $X$ must also be bounded.) On the other hand, there are nonzero Radon measures in $N_{0}^{1, p}(\Omega)^{\prime}$, so the existence of a corresponding supersolution fails.

Proof of Proposition 5.1. In [20, Propositions 3.5 and 3.9], the result is stated under stronger assumptions than here, but the proof of this result is valid under our assumptions. In particular, since $C_{p}(X \backslash \Omega)>0$, the coercivity of the map $T$ follows from the Poincaré inequality for $N_{0}^{1, p}$ (also called the $p$-Friedrichs inequality), whose proof can be found, e.g., in [9, Corollary 5.54].

In [20], the uniqueness was shown up to equivalence between supersolutions. The pointwise uniqueness for lsc-regularized supersolutions then follows from (4.1). That $u$ is nonnegative follows from Lemma 5.3 below, as $u \equiv 0$ is the lsc-regularized supersolution corresponding to the zero measure.

We need the following comparison principle.

Lemma 5.3. Let $v_{1}, v_{2} \in N_{0}^{1, p}(\Omega)^{\prime}$ be Radon measures such that $v_{1} \leq v_{2}$. If $u_{1}, u_{2} \in N_{0}^{1, p}(\Omega)$ are the corresponding lsc-regularized supersolutions given by Proposition 5.1, then $u_{1} \leq u_{2}$ on $\Omega$. 
Proof. Inserting $\varphi=\left(u_{1}-u_{2}\right)_{+} \in N_{0}^{1, p}(\Omega)$ into (5.1) for $u_{1}$ and $u_{2}$ gives

$$
\begin{aligned}
0 & \leq \int_{\Omega} \varphi d \nu_{2}-\int_{\Omega} \varphi d \nu_{1}=\int_{\Omega}\left(\left|D u_{2}\right|^{p-2} D u_{2}-\left|D u_{1}\right|^{p-2} D u_{1}\right) \cdot D \varphi d \mu \\
& =\int_{\left\{u_{1}>u_{2}\right\}}\left(\left|D u_{2}\right|^{p-2} D u_{2}-\left|D u_{1}\right|^{p-2} D u_{1}\right) \cdot\left(D u_{1}-D u_{2}\right) d \mu .
\end{aligned}
$$

Since $\xi \mapsto|\xi|^{p-2} \xi$ is strictly monotone, the integrand is nonpositive. Thus $D u_{1}(x)=D u_{2}(x)$ for a.e. $x$ such that $u_{1}(x)>u_{2}(x)$, and hence $D \varphi=0$ a.e. in $\Omega$. The Poincaré inequality for $N_{0}^{1, p}$ (e.g. [9, Corollary 5.54]) then yields

$$
\int_{\Omega} \varphi^{p} d \mu \leq C_{\Omega} \int_{\Omega}|D \varphi|^{p} d \mu=0
$$

Hence $\varphi=0$ a.e. in $\Omega$, i.e. $u_{1} \leq u_{2}$ a.e. on $\Omega$. As $u_{1}$ and $u_{2}$ are lsc-regularized, $u_{1} \leq u_{2}$ everywhere in $\Omega$.

Definition 5.4. Let $u_{E}$ be the Cheeger capacitary potential of $E$ in $\Omega$, given by Definition 4.4. Then $v_{E}=T u_{E}$ is the capacitary measure of $E$ on $\Omega$, where $T$ is the operator defined by (5.1).

Remark 5.5. Note that the Cheeger capacitary potential $u_{E}$ is the lsc-regularized solution of the $\mathcal{K}_{\psi, 0}(\Omega)$-Cheeger obstacle problem, where $\psi=1$ on $E$ and $\psi=-\infty$ otherwise. Hence, for every $\varphi \in N_{0}^{1, p}(\Omega \backslash E)$ and every $t>0$, the function $u_{E}+t \varphi \in \mathcal{K}_{\psi, 0}(\Omega)$ and thus

$$
0 \leq \int_{\Omega}\left(\left|D u_{E}+t D \varphi\right|^{p}-\left|D u_{E}\right|^{p}\right) d \mu .
$$

Dividing by $t$ and letting $t \rightarrow 0$ shows that

$$
\int_{\Omega}\left|D u_{E}\right|^{p-2} D u_{E} \cdot D \varphi d \mu \geq 0
$$

see $[48,(2.8)]$. Applying this also to $-\varphi$ shows that equality must hold in (5.3). Consequently, the capacitary measure $v_{E}=T u_{E}$ satisfies

$$
\int_{\Omega} \varphi d v_{E}=0 \quad \text { for every } \varphi \in N_{0}^{1, p}(\Omega \backslash E) .
$$

We need the following lemma when proving the Cartan property (Theorem 1.1). Later, in Theorem 9.1, we generalize this lemma to quasiopen sets and, as a consequence, obtain that the capacitary measure $v_{E}$ is supported on the fine boundary $\partial_{p} E$; that it is supported on the boundary $\partial E$ is well known. 
Lemma 5.6. Let $E \subset \Omega$ be such that $\operatorname{cap}_{p}(E, \Omega)<\infty$, and let $u_{E}$ be the Cheeger capacitary potential of $E$ on $\Omega$ and $v_{E}=T u_{E}$ be the corresponding capacitary measure. If $G \subset \Omega$ is open and $v \in N^{1, p}(\Omega)$ is bounded and such that $v=1$ q.e. on $G \cap E$, then

$$
\int_{G} v d v_{E}=\int_{G} u_{E} d v_{E}
$$

In particular, $v_{E}(G)=\int_{G} u_{E} d \nu_{E}$; and if $C_{p}(G \cap E)=0$, then $v_{E}(G)=0$. Moreover, $v_{E}(\Omega)=\operatorname{Ch}_{-} \operatorname{cap}_{p}(E, \Omega)$.

Proof. For every $\eta \in \operatorname{Lip}_{c}(G)$ with $0 \leq \eta \leq 1, \eta\left(v-u_{E}\right) \in N_{0}^{1, p}(\Omega \backslash E)$. Thus, (5.4) yields

$$
\int_{G} \eta\left(v-u_{E}\right) d v_{E}=0
$$

Since $v-u_{E}$ and $G$ are bounded, dominated convergence and letting $\eta \nearrow \chi_{G}$ imply (5.5). For the second statement, apply this to $v=1$ and $v=0$, respectively. The last identity is obtained by inserting $\varphi=u_{E}$ into (5.1).

\section{Thinness and the fine topology}

We now define the fine topological notions which are central in this paper.

Definition 6.1. A set $E \subset X$ is thin at $x \in X$ if

$$
\int_{0}^{1}\left(\frac{\operatorname{cap}_{p}(E \cap B(x, r), B(x, 2 r))}{\operatorname{cap}_{p}(B(x, r), B(x, 2 r))}\right)^{1 /(p-1)} \frac{d r}{r}<\infty .
$$

A set $U \subset X$ is finely open if $X \backslash U$ is thin at each point $x \in U$.

It is easy to see that the finely open sets give rise to a topology, called the fine topology. Every open set is finely open, but the converse is not true in general.

In the definition of thinness, we make the convention that the integrand is 1 whenever $\operatorname{cap}_{p}(B(x, r), B(x, 2 r))=0$. This happens, e.g., if $X=B(x, 2 r)$, but never if $r<\frac{1}{2} \operatorname{diam} X$. Note that thinness is a local property. Because of (4.3), thinness can equivalently be defined using the Cheeger variational capacity Ch-cap ${ }_{p}$.

Definition 6.2. A function $u: U \rightarrow \overline{\mathbf{R}}$, defined on a finely open set $U$, is finely continuous if it is continuous when $U$ is equipped with the fine topology and $\overline{\mathbf{R}}$ with the usual topology.

Since every open set is finely open, the fine topology generated by the finely open sets is finer than the metric topology. By [19, Theorem 4.4], [44, Theorem 4.3] and [11, Theorem 1.1], it is the coarsest topology making all (Cheeger) 
superharmonic functions finely continuous. See [9, Section 11.6] and [11] for further discussion on thinness and the fine topology.

\section{The Cartan, Choquet and Kellogg properties}

We start this section by proving the Cartan property (Theorem 1.1). The proof combines arguments in [40, p. 155] with those in [48, Section 2.1.5]. As in [40], the pointwise estimate (7.1) is essential here. However, in [40], to obtain the estimate $v_{k}\left(B_{j}\right) \leq \operatorname{cap}\left(E_{j}, B_{j-1}\right)$, the authors used the dual characterization of capacity as the supremum of measures on $E_{j}$ with potentials bounded by 1 . A similar estimate also follows from [48, Theorem 2.45]. Here, we instead use a direct derivation of $v_{k}\left(B_{j}\right) \leq \operatorname{cap}\left(E_{j}, B_{j-1}\right)$ based on (5.1), Remark 5.5, and Lemma 5.6.

Proof of Theorem 1.1. By [11, Lemma 4.7], we may assume that $E$ is open. Let $B_{j}=B\left(x_{0}, r_{j}\right), r_{j}=2^{-j}, E_{j}=E \cap B_{j}$, and $u_{j}$ be the Cheeger capacitary potential of $E_{j}$ with respect to $B_{j-1}, j=1,2, \ldots$. As $E_{j}$ is open, $u_{j}=1$ in $E_{j}$. Let $k \geq 1$ be an integer to be specified later but so large that diam $B_{k}<\frac{1}{6} \operatorname{diam} X$, and let $v_{k}=T u_{k}$ be the Radon measure in $N_{0}^{1, p}\left(B_{k-1}\right)^{\prime}$, given by Proposition 5.1.

Since $u_{k}=1$ in $E_{k}$, it remains to show that $u_{k}\left(x_{0}\right)<1$ for some $k$. By [42, Remark 5.4] (or [9, Proposition 8.24]), $x_{0}$ is a Lebesgue point of $u_{k}$. Hence, [20, Proposition 4.10] shows that

$$
u_{k}\left(x_{0}\right) \leq c\left(f_{B_{k}} u_{k}^{p} d \mu\right)^{1 / p}+c \sum_{j=k-1}^{\infty}\left(r_{j}^{p} \frac{\nu_{k}\left(B_{j}\right)}{\mu\left(B_{j}\right)}\right)^{1 /(p-1)} .
$$

The first term on the right-hand side can be estimated using the Sobolev inequality [9, Theorem 5.51] and the fact that $\operatorname{cap}_{p}\left(B_{k}, B_{k-1}\right) \simeq r_{k}^{-p} \mu\left(B_{k}\right)$ (by [17, Lemma 3.3] or [9, Proposition 6.16]) as

$$
f_{B_{k}} u_{k}^{p} d \mu \leq \frac{1}{\mu\left(B_{k}\right)} \int_{B_{k-1}} u_{k}^{p} d \mu \leq \frac{C r_{k}^{p}}{\mu\left(B_{k}\right)} \int_{B_{k-1}}\left|D u_{k}\right|^{p} d \mu \simeq \frac{\operatorname{cap}_{p}\left(E_{k}, B_{k-1}\right)}{\operatorname{cap}_{p}\left(B_{k}, B_{k-1}\right)}
$$

Here we have also used (4.2) and (4.3).

As for the second term in (7.1), let $v_{j}$ be the lsc-regularized solution of $T v_{j}=$ $\left.v_{k}\right|_{B_{j}}$ in $B_{k-1}, j \geq k$. Lemma 5.3 shows that $v_{j} \leq u_{k} \leq 1$ in $B_{k-1}$. Thus, with $v_{j}$ as a test function in (5.1), we have

$$
\int_{B_{k-1}}\left|D v_{j}\right|^{p} d \mu=\int_{B_{j}} v_{j} d v_{k} \leq \int_{B_{j}} u_{k} d v_{k}
$$

Using Lemma 5.6 (for the first equality below) and (5.1) with $u_{j}$ as a test function 
(for the third equality), we obtain

$$
\begin{aligned}
\int_{B_{j}} u_{k} d v_{k} & =\int_{B_{j}} u_{j} d v_{k}=\left.\int_{B_{k-1}} u_{j} d v_{k}\right|_{B_{j}}=\int_{B_{k-1}}\left|D v_{j}\right|^{p-2} D v_{j} \cdot D u_{j} d \mu \\
& \leq\left(\int_{B_{k-1}}\left|D v_{j}\right|^{p} d \mu\right)^{1-1 / p}\left(\int_{B_{k-1}}\left|D u_{j}\right|^{p} d \mu\right)^{1 / p} .
\end{aligned}
$$

Together with (7.3), this implies that

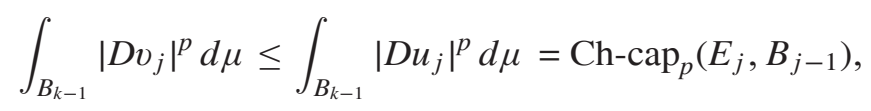

where Ch-cap ${ }_{p}$ denotes the Cheeger variational capacity. Inserting this into (7.4) yields $\int_{B_{j}} u_{k} d v_{k} \leq \mathrm{Ch}-\operatorname{cap}_{p}\left(E_{j}, B_{j-1}\right)$ which, together with the last part of Lemma 5.6 and (4.3), shows that

$$
v_{k}\left(B_{j}\right)=\int_{B_{j}} u_{k} d v_{k} \leq \mathrm{Ch}-\operatorname{cap}_{p}\left(E_{j}, B_{j-1}\right) \simeq \operatorname{cap}_{p}\left(E_{j}, B_{j-1}\right) .
$$

Hence, using $\operatorname{cap}_{p}\left(B_{j}, B_{j-1}\right) \simeq r_{j}^{-p} \mu\left(B_{j}\right)$ again, we obtain

$$
\sum_{j=k-1}^{\infty}\left(r_{j}^{p} \frac{\nu_{k}\left(B_{j}\right)}{\mu\left(B_{j}\right)}\right)^{1 /(p-1)} \leq C \sum_{j=k-1}^{\infty}\left(\frac{\operatorname{cap}_{p}\left(E_{j}, B_{j-1}\right)}{\operatorname{cap}_{p}\left(B_{j}, B_{j-1}\right)}\right)^{1 /(p-1)} .
$$

Since $E$ is thin at $x_{0}$, both (7.2) and (7.5) can be made arbitrarily small by choosing $k$ large enough. Thus $u_{k}\left(x_{0}\right)<1$ for large enough $k$.

We now turn to the proof of the Choquet property (Theorem 1.2). The following notation is common in the literature. The base $b_{p} E$ of a set $E \subset X$ consists of all points $x \in X$ at which $E$ is thick, i.e., not thin. Using this notation, we can formulate the Choquet property as follows.

Theorem 7.1 (Choquet property). For each $E \subset X$ and $\varepsilon>0$, there exists an open set $G$ such that $G \cup b_{p} E=X$ and $C_{p}(E \cap G)<\varepsilon$.

Proof. Let $\left\{B_{j}\right\}_{j=1}^{\infty}$ be a countable covering of $X$ by balls such that every point is covered by arbitrarily small balls. Such a covering exists as $X$ is separable. Choose $\varepsilon>0$. For each $j$, let $u_{j}$ be the Cheeger capacitary potential of $E \cap B_{j}$ with respect to $2 B_{j}$. Since each $u_{j}$ is quasicontinuous, there is an open set $G_{j}^{\prime}$ with $C_{p}\left(G_{j}^{\prime}\right)<2^{-j} \varepsilon$ such that the set

$$
G_{j}:=\left\{x \in B_{j}: u_{j}(x)<1\right\} \cup G_{j}^{\prime}
$$

is open. We set $G:=\bigcup_{j=1}^{\infty} G_{j}$ and show that $G \cup b_{p} E=X$. 
Choose $z \in X \backslash b_{p} E$. If $\operatorname{dist}(z, E \backslash\{z\})>0$, there exists $B_{j} \ni z$ such that $B_{j} \cap E=\varnothing$ or $B_{j} \cap E=\{z\}$. If $B_{j} \cap E=\varnothing$, then $u_{j} \equiv 0$. If $B_{j} \cap E=\{z\}$, then the thinness of $E$ at $z$, together with [11, Proposition 1.3], shows that $C_{p}(\{z\})=0$, and hence $u_{j} \equiv 0$. In both cases, $z \in B_{j} \subset G_{j} \subset G$.

We can therefore assume that $z \in \overline{E \backslash\{z\}}$. By Theorem 1.1 (applied to $E \backslash\{z\})$, there is a bounded positive Cheeger superharmonic function $v$ in an open neighbourhood of $z$ such that $v(z)<1<\liminf _{E \ni x \rightarrow z} v(x)$. Hence we may fix a ball $B_{j} \ni z$ such that $v$ is Cheeger superharmonic in $3 B_{j}$ and $v \geq 1$ in $B_{j} \cap E$. Since $v$ is the lsc-regularized solution of the $\mathcal{K}_{v, v}\left(2 B_{j}\right)$-Cheeger obstacle problem and $u_{j}$ is the lsc-regularized solution of the $\mathcal{K}_{\chi_{B_{j} \cap E}, 0}\left(2 B_{j}\right)$-Cheeger obstacle problem, the comparison principle [8, Lemma 5.4] (or [9, Lemma 8.30]) yields $u_{j} \leq v$ in $2 B_{j}$. It follows that $u_{j}(z)<1$, and thus $z \in G_{j} \subset G$.

It remains to prove that $C_{p}(E \cap G)<\varepsilon$. For any $j$, we have $u_{j} \geq 1$ q.e. in $E \cap B_{j}$, and thus (7.6) implies

$$
C_{p}\left(E \cap G_{j}\right) \leq C_{p}\left(\left\{x \in E \cap B_{j}: u_{j}(x)<1\right\}\right)+C_{p}\left(G_{j}^{\prime}\right)=C_{p}\left(G_{j}^{\prime}\right)<2^{-j} \varepsilon .
$$

By the countable subadditivity of the capacity, we obtain $C_{p}(E \cap G)<\varepsilon$.

We can now deduce Corollary 1.3 as a consequence of the Choquet property.

Corollary 7.2 (Fine Kellogg property). For every $E \subset X$,

$$
C_{p}\left(E \backslash b_{p} E\right)=0 .
$$

Proof. For every $\varepsilon>0$, Theorem 7.1 provides us with an open set $G$ such that $G \cup b_{p} E=X$ and $C_{p}(E \cap G)<\varepsilon$. Then $E \backslash b_{p} E \subset E \cap G$, and therefore $C_{p}\left(E \backslash b_{p} E\right)<\varepsilon$. Letting $\varepsilon \rightarrow 0$ concludes the proof.

Remark 7.3. Let $\Omega \subset X$ be a bounded open set with $C_{p}(X \backslash \Omega)>0$. Choosing $E=X \backslash \Omega$ in Corollary 7.2 gives

$$
C_{p}\left(\partial \Omega \backslash b_{p}(X \backslash \Omega)\right) \leq C_{p}\left((X \backslash \Omega) \backslash b_{p}(X \backslash \Omega)\right)=0 .
$$

On the other hand, a boundary point $x_{0} \in \partial \Omega$ is regular (both for $p$-harmonic functions defined through upper gradients and for Cheeger $p$-harmonic functions) whenever $X \backslash \Omega$ is thick at $x_{0}$, by the sufficiency part of the Wiener criterion; see [20], [18], and [19] (or [9, Theorem 11.24]). Hence (7.7) yields that the set of irregular boundary points of $\Omega$ is of capacity zero. This result was obtained by a different method (and called the Kellogg property) in [14, Theorem 3.9]. Thus it is quite natrual to call Corollary 7.2 the Kellogg property. 
To clarify that the above proof of the Kellogg property is not using circular reasoning, let us explain how the results we use here are obtained in [9]. Here, we need only results up to Chapter 9 and the results in Sections 11.4 and 11.6. They, in turn, rely only on results up to Chapter 9 and the implication (b) $\Rightarrow$ (a) in Theorem 10.29, which can easily be obtained just using comparison. Hence we are not relying on the Kellogg property obtained in [9, Section 10.2].

\section{Finely open and quasiopen sets}

We start this section by using the Choquet property to prove Theorem 1.4; i.e., we characterize quasiopen sets and quasicontinuity by means of the corresponding fine topological notions. We then give several immediate applications of this characterization.

Note that if $C_{p}(\{x\})=0$, then $\{x\}$ is quasiopen, but not finely open. Thus the zero capacity set in Theorem 1.4(a) cannot be dropped.

Proof of Theorem 1.4. (a) That each quasiopen set $U$ is of the form $U=$ $V \cup E$ for some finely open set $V$ and for a set $E$ of capacity zero, was recently shown in [11, Theorem 4.9].

For the converse, assume that $U=V \cup E$, where $V$ is finely open and $C_{p}(E)=$ 0 . Let $\varepsilon>0$. By the Choquet property (Theorem 7.1), applied to $X \backslash V$, there is an open set $G$ such that $G \cup b_{p}(X \backslash V)=X$ and $C_{p}(G \backslash V)<\varepsilon$. The capacity $C_{p}$ is an outer capacity, by [15, Corollary 1.3] (or [9, Theorem 5.31]), so there is an open set $\widetilde{G} \supset(G \backslash V) \cup E$ such that $C_{p}(\widetilde{G})<\varepsilon$. Since $V$ is finely open, we have $V \subset X \backslash b_{p}(X \backslash V) \subset G$, and thus $U \cup \widetilde{G}=V \cup \widetilde{G}=G \cup \widetilde{G}$ is open, i.e. $U$ is quasiopen.

(b) If $u$ is quasicontinuous, then it is finite q.e., by definition, and finely continuous q.e., by [11, Theorem 4.9].

Conversely, assume that there is a set $Z$ with $C_{p}(Z)=0$ such that $u$ is finite and finely continuous on $V:=U \backslash Z$. By (a), we can assume that $V$ is finely open. Let $\varepsilon>0$, let $\left\{\left(a_{j}, b_{j}\right)\right\}_{j=1}^{\infty}$ be an enumeration of all open intervals with rational endpoints, and set $V_{j}:=\left\{x \in V: a_{j}<u(x)<b_{j}\right\}$. By the fine continuity of $u$, the sets $V_{j}$ are finely open. Hence by (a), $V_{j}$ are quasiopen, and thus there are open sets $G_{j}$ and $G_{U}$ with $C_{p}\left(G_{j}\right)<2^{-j} \varepsilon$ and $C_{p}\left(G_{U}\right)<\varepsilon$ such that $V_{j} \cup G_{j}$ and $U \cup G_{U}$ are open. Also, as $C_{p}$ is an outer capacity, there is an open set $G_{Z} \supset Z$ with $C_{p}\left(G_{Z}\right)<\varepsilon$. Then $G:=G_{Z} \cup G_{U} \cup \bigcup_{j=1}^{\infty} G_{j}$ is open, $C_{p}(G)<3 \varepsilon$, and $\left.u\right|_{U \backslash G}$ is continuous, since $V_{j} \cup G$ are open sets. 
Theorem 1.4 leads directly to the following improvements of the results in [10]. A set $U$ is $p$-path open if for $p$-almost every curve $\gamma:\left[0, l_{\gamma}\right] \rightarrow X$, the set $\gamma^{-1}(U)$ is (relatively) open in $\left[0, l_{\gamma}\right]$.

Corollary 8.1. Every finely open set is quasiopen, measurable, and p-path open.

Proof. By Theorem 1.4(a), every finely open set is quasiopen. Hence the result follows from [51, Remark 3.5] and [10, Lemma 9.3].

An important consequence is that the restriction of a minimal $p$-weak upper gradient to a finely open set remains minimal. This was shown for measurable $p$ path open sets in [10, Corollary 3.7]. We restate this result in view of Corollary 8.1. In order to do so in full generality, we need to introduce some more notation.

We define the Dirichlet space

$$
D^{p}(X)=\left\{u: u \text { is measurable and has an upper gradient in } L^{p}(X)\right\} .
$$

As with $N^{1, p}(X)$, we assume that functions in $D^{p}(X)$ are defined everywhere (with values in $\overline{\mathbf{R}}:=[-\infty, \infty])$. For a measurable set $E \subset X$, the spaces $D^{p}(E)$ and $D_{\text {loc }}^{p}(E)$ are defined similarly. For $u \in D_{\text {loc }}^{p}(E)$, we denote the minimal $p$-weak upper gradient of $u$ taken with $E$ as the underlying space by $g_{u, E}$. Its existence is guaranteed by [9, Theorem 2.25].

Corollary 8.2. Let $U$ be quasiopen and $u \in D_{\mathrm{loc}}^{p}(X)$. Then $g_{u, U}=g_{u}$ a.e. in $U$. In particular, $g_{u, U}=g_{u}$ a.e. in $U$ if $U$ is finely open.

Proof. By [51, Remark 3.5] and [10, Lemma 9.3], every quasiopen set is $p$ path open and measurable, whereas Theorem 1.4(a) shows that every finely open set is quasiopen. Hence the result follows from [10, Corollary 3.7].

In [10], the fine topology turned out to be important for analyzing obstacle problems on nonopen measurable sets, i.e., when minimizing the $p$-energy integral

$$
\int_{E} g_{u, E} d \mu
$$

on an arbitrary bounded measurable set $E$ among all functions

$$
u \in \mathcal{K}_{\psi_{1}, \psi_{2}, f}(E):=\left\{v \in D^{p}(E): v-f \in N_{0}^{1, p}(E) \text { and } \psi_{1} \leq v \leq \psi_{2} \text { q.e. in } E\right\} .
$$

Knowing that finely open sets are measurable and $p$-path open, we are now able to improve and simplify some of the results therein. We summarize these improvements in the following theorem, which follows directly from [10, Theorems 1.2 and 8.3, and Corollaries 3.7 and 7.4] and Corollary 8.1. We denote the fine interior of $E$ by fine-int $E$. 
Theorem 8.3. Let $E \subset X$ be a bounded measurable set such that $C_{p}(X \backslash E)>0$, and let $f \in D^{p}(E)$ and $\psi_{j}: E \rightarrow \overline{\mathbf{R}}, j=1,2$, be such that $\mathcal{K}_{\psi_{1}, \psi_{2}, f}(E) \neq \varnothing$. Also let $E_{0}=$ fine-int $E$. Then $\mathcal{K}_{\psi_{1}, \psi_{2}, f}(E)=\mathcal{K}_{\psi_{1}, \psi_{2}, f}\left(E_{0}\right)$, and the solutions of the minimization problem for $(8.1)$ with respect to $\mathcal{K}_{\psi_{1}, \psi_{2}, f}(E)$ and $\mathcal{K}_{\psi_{1}, \psi_{2}, f}\left(E_{0}\right)$ coincide. Moreover, $g_{u, E_{0}}=g_{u, E}$ a.e. in $E_{0}$; and if $\mu\left(E \backslash E_{0}\right)=0$, then the p-energies associated with these two minimization problems also coincide. Furthermore, if $f \in D^{p}(\Omega)$ for some open set $\Omega \supset E$, then $g_{u, E_{0}}=g_{u, E}=g_{u}$ a.e. in $E_{0}$ and the above solutions coincide with the solutions of the corresponding $\mathcal{K}_{\psi_{1}^{\prime}, \psi_{2}^{\prime}, f}(\Omega)$-obstacle problem, where $\psi_{j}^{\prime}$ is the extension of $\psi_{j}$ to $\Omega \backslash E$ by $f$, $j=1,2$.

We also obtain the following consequence of [10, Lemma 3.9 and Theorem 7.3], which generalizes [48, Theorem 2.147 and Corollary 2.162] to metric spaces and to arbitrary sets; see also [48, Remark 2.148] for another description of $W_{0}^{1, p}(\Omega)$ in $\mathbf{R}^{n}$.

Proposition 8.4 ( cf. [10, Proposition 9.4]). Let $E \subset X$ be arbitrary and $u \in N^{1, p}\left(\bar{E}^{p}\right)$, where $\bar{E}^{p}$ is the fine closure of $E$. Then $u \in N_{0}^{1, p}(E)$ if and only if $u=0$ q.e. on the fine boundary $\partial_{p} E:=\bar{E}^{p} \backslash$ fine-int $E$ of $E$.

\section{Support of capacitary measures}

We can now bootstrap Lemma 5.6 to quasiopen sets and, in particular, show that the capacitary measure $\nu_{E}$ only charges the fine boundary $\partial_{p} E:=\bar{E}^{p} \backslash$ fine-int $E$ of $E$, where $\bar{E}^{p}$ is the fine closure of $E$. This observation seems to be new, even in unweighted $\mathbf{R}^{n}$; see also Corollary 9.6.

Theorem 9.1. Let $\Omega$ be a nonempty bounded open set with $C_{p}(X \backslash \Omega)>0$. Let $E \subset \Omega, u_{E}$ and $v_{E}=T u_{E}$ be as in Lemma 5.6. Let $U \subset \Omega$ be quasiopen and $v \in N^{1, p}(\Omega)$.

(a) If $u \in N^{1, p}(\Omega)$ and either $u$ is bounded from below or belongs to $L^{1}\left(v_{E}\right)$, and $u=v$ q.e. in $U \cap E$, then

$$
\int_{U} u d v_{E}=\int_{U} v d v_{E}
$$

(b) If $v=1$ q.e. in $U \cap E$, then

$$
v_{E}(U)=\int_{U} v d v_{E}=\int_{U} u_{E} d v_{E} .
$$

(c) If $C_{p}(U \cap E)=0$, then $v_{E}(U)=0$. 
To prove Theorem 9.1 we need the following quasi-Lindelöf principle, whose proof in unweighted $\mathbf{R}^{n}$ is given in [35, Theorem 2.3]. The proof there, which relies on the fine Kellogg property, extends to metric spaces; see [12].

Theorem 9.2 (Quasi-Lindelöf principle). For each family $V$ of finely open sets, there is a countable subfamily $V^{\prime}$ such that

$$
C_{p}\left(\bigcup_{V \in \mathcal{V}} V \backslash \bigcup_{V^{\prime} \in \mathcal{V}^{\prime}} V^{\prime}\right)=0 .
$$

We also need the following lemmas.

Lemma 9.3. Let $U$ be finely open, and let $x_{0} \in U$. Then there exists a finely open set $V \Subset U$ containing $x_{0}$ and a function $v \in N_{0}^{1, p}(U)$ such that $v=1$ on $V$ and $0 \leq v \leq 1$ everywhere.

Proof. Since $U$ is finely open, $E:=X \backslash U$ is thin at $x_{0}$. By the Cartan property (Theorem 1.1), there are a ball $B \ni x_{0}$ and a lower semicontinuous finely continuous $u \in N^{1, p}(B)$ such that $0 \leq u \leq 1$ in $B, u\left(x_{0}\right)<1$ and $u=1$ on $E \cap B$. Let $\eta \in \operatorname{Lip}_{c}(B)$ be such that $0 \leq \eta \leq 1$ on $B$ and $\eta=1$ on $\frac{1}{2} B$. Then $w:=\eta(1-u) \in N_{0}^{1, p}(U)$ is upper semicontinuous and finely continuous on $X$, and $w\left(x_{0}\right)=1-u\left(x_{0}\right)>0$. Let $v=\min \left\{1,2 w / w\left(x_{0}\right)\right\} \in N_{0}^{1, p}(U)$ and $V=\left\{x \in U: w(x)>\frac{1}{2} w\left(x_{0}\right)\right\}$. The fine continuity and upper semicontinuity of $w$ imply that $V$ is finely open and $V \Subset U$. Moreover, $x_{0} \in V$ and $v=1$ on $V$.

Lemma 9.4. Let $U \subset X$ be quasiopen. Then

$$
U=W_{1} \cup E_{1}=W_{2} \backslash E_{2},
$$

where $W_{1}$ and $W_{2}$ are Borel sets and $E_{1}$ and $E_{2}$ are of capacity zero. Moreover, we may choose $W_{1}$ to be of type $F_{\sigma}$ and $W_{2}$ to be of type $G_{\delta}$.

Not all finely open sets are Borel. For instance, $V=G \backslash A$, where $G$ is open and $A \subset G$ is a non-Borel set with $C_{p}(A)=0$, is a non-Borel finely open set. To be more specific, we may let $A \subset G \subset \mathbf{R}^{n}$ be any non-Borel set of Hausdorff dimension $<n-p$.

Proof. By definition, for each $j=1,2, \ldots$, there is an open set $G_{j}$ such that $U \cup G_{j}$ is open and $C_{p}\left(G_{j}\right)<1 / j$. Then

$$
\begin{aligned}
U & =\left(U \backslash \bigcap_{j=1}^{\infty} G_{j}\right) \cup\left(U \cap \bigcap_{j=1}^{\infty} G_{j}\right)=\bigcup_{j=1}^{\infty}\left(U \backslash G_{j}\right) \cup \bigcap_{j=1}^{\infty}\left(U \cap G_{j}\right) \\
& =\bigcup_{j=1}^{\infty}\left(\left(U \cup G_{j}\right) \backslash G_{j}\right) \cup \bigcap_{j=1}^{\infty}\left(U \cap G_{j}\right)=: W_{1} \cup E_{1} .
\end{aligned}
$$


The second equality in (9.2) follows by choosing $W_{2}=\bigcap_{j=1}^{\infty}\left(U \cup G_{j}\right)$ and $E_{2}=$ $W_{2} \backslash U$. The last two claims follow from the choices above.

Proof of Theorem 9.1. By Theorem 1.4, we can find a finely open set $V \subset U$ such that $C_{p}(U \backslash V)=0$. For every $x \in V$, Lemma 9.3 provides us with a finely open set $V_{x} \Subset V$ containing $x$ and a function $v_{x} \in N_{0}^{1, p}(V)$ such that $v_{x}=1$ on $V_{x}$ and $0 \leq v_{x} \leq 1$ everywhere. By the quasi-Lindelöf principle and the fact that $C_{p}(U \backslash V)=0$, we can choose out of these $V_{j}=V_{x_{j}}$ and $v_{j}=v_{x_{j}}$, $j=1,2, \ldots$, such that $U=\bigcup_{j=1}^{\infty} V_{j} \cup Z$, where $C_{p}(Z)=0$. For $k=1,2, \ldots$, set

$$
\eta_{k}=\chi_{X \backslash Z} \max _{j=1,2, \ldots, k} v_{j} \in N_{0}^{1, p}(U) .
$$

Since $v_{E}$ is a complete Borel measure which, by Lemma 5.6 (or [20, Lemma 3.8]), is absolutely continuous with respect to the capacity $C_{p}$, it follows from Lemma 9.4 that $U$ is $v_{E}$-measurable and $v_{E}(Z)=0$. We are now ready to prove (a)-(c).

(a) First assume that $u$ and $v$ are bounded. Then $\eta_{k}(u-v) \in N^{1, p}(U)$, by Lemma 2.6, and [9, Lemma 2.37] shows that $\eta_{k}(u-v) \in N_{0}^{1, p}(U)$. Since $u=v$ q.e. on $U \cap E$, it follows that $\eta_{k}(u-v) \in N_{0}^{1, p}(U \backslash E)$. Hence (5.4) yields that

$$
\int_{U} \eta_{k}(u-v) d v_{E}=0 \text {. }
$$

Since $\eta_{k} \nearrow \chi_{U \backslash Z}$ on $U$, dominated convergence and the fact that $v_{E}(Z)=0$ imply that

$$
\int_{U}(u-v) d v_{E}=\int_{U \backslash Z}(u-v) d \nu_{E}=0,
$$

and (9.1) follows.

Next, assume that $u$ and $v$ are bounded from below. Then, by monotone convergence and the bounded case,

$$
\int_{U} u d v_{E}=\lim _{k \rightarrow \infty} \int_{U} \min \{u, k\} d v_{E}=\lim _{k \rightarrow \infty} \int_{U} \min \{v, k\} d v_{E}=\int_{U} v d v_{E} .
$$

Finally, applying this to the positive and negative parts of $u$ and $v$ gives

$$
\int_{U} u_{+} d v_{E}=\int_{U} v_{+} d v_{E} \text { and } \int_{U} u_{-} d v_{E}=\int_{U} v_{-} d v_{E},
$$

and hence

$$
\int_{U} u d v_{E}=\int_{U} u_{+} d v_{E}-\int_{U} u_{-} d v_{E}=\int_{U} v_{+} d v_{E}-\int_{U} v_{-} d v_{E}=\int_{U} v d v_{E},
$$

where the assumptions on $u$ guarantee that the subtractions are well-defined (i.e., not $\infty-\infty)$. 
(b) Applying a to $u=u_{E}$ and $v$, we have $\int_{U} v d v_{E}=\int_{U} u_{E} d v_{E}$. Choosing $v \equiv 1$ yields $v_{E}(U)=\int_{U} u_{E} d v_{E}$.

(c) This follows by applying b to $v \equiv 0$.

Corollary 9.5. Let $\Omega, E, u_{E}$ and $v_{E}$ be as in Theorem 9.1. Then

$$
\nu_{E}\left(\Omega \backslash \partial_{p} E\right)=0,
$$

i.e. $v_{E}$ is supported on the fine boundary $\partial_{p} E:=\bar{E}^{p} \backslash$ fine-int $E$ of $E$.

Proof. First, observe that the fine exterior $V=\Omega \backslash \bar{E}^{p}$ is finely open and $V \cap E=\varnothing$, whence $v_{E}(V)=0$ by Theorem 9.1(c).

Next, observe that the fine interior $E_{0}:=$ fine-int $E$ is finely open, and, as in the proof of Theorem 9.1, we can use the quasi-Lindelöf principle to find nonnegative $\eta_{k} \in N_{0}^{1, p}\left(E_{0}\right)$ such that

$$
\eta_{k} \nearrow \chi_{E_{0} \backslash Z} \text { as } k \rightarrow \infty
$$

where $C_{p}(Z)=0$. Since $u_{E}=1$ q.e. in $E$, we have $D u_{E}=0$ a.e. in $E$; and hence, by (5.1),

$$
\int_{\Omega} \eta_{k} d v_{E}=\int_{\Omega}\left|D u_{E}\right|^{p-2} D u_{E} \cdot D \eta_{k} d \mu=0
$$

Dominated convergence then shows that $v_{E}\left(E_{0} \backslash Z\right)=0$. Since $v_{E}(Z)=0$ by Lemma 5.6 (or [20, Lemma 3.8]), the proof is complete.

Using Corollary 9.5, we can now obtain the following result, similar to Theorem 9.1, but with $U \cap E$ replaced by $U \cap \partial_{p} E$.

Corollary 9.6. Let $\Omega, E \subset \Omega, u_{E}$ and $v_{E}=T u_{E}$ be as in Lemma 5.6. Let $U \subset \Omega$ be quasiopen.

(a) If $u$ is a function on $\Omega$ such that $\int_{U \cap \partial_{p} E} u d \nu_{E}$ is well-defined and $v$ is a function on $U$ such that $v=u$ q.e. in $U \cap \partial_{p} E$, then

$$
\int_{U} v d v_{E}=\int_{U} u d v_{E} .
$$

(b) If $v=1$ q.e. in $U \cap \partial_{p} E$, then

$$
v_{E}(U)=\int_{U} v d v_{E}=\int_{U} u_{E} d v_{E}
$$

(c) If $C_{p}\left(U \cap \partial_{p} E\right)=0$, then $v_{E}(U)=0$.

Proof. (c) This follows directly from Corollary 9.5 and the fact that $v_{E}$ is absolutely continuous with respect to the capacity $C_{p}$ (by Lemma 5.6). 
(a) By Corollary 9.5 and the absolute continuity of $v_{E}$ with respect to $C_{p}$ again, we see that

$$
\int_{U} v d v_{E}=\int_{U \cap \partial_{p} E} v d v_{E}=\int_{U \cap \partial_{p} E} u d v_{E}=\int_{U} u d v_{E}
$$

(b) This follows from (a), by choosing $u \equiv 1$ and $u=u_{E}$, respectively.

We conclude this paper with a simple example showing that the fine boundary can be much smaller than the metric boundary. A much more involved example in the same spirit is given in [10, Section 9].

Example 9.7. Let $B$ be an open ball in $\mathbf{R}^{n}, 1<p \leq n$, and let $E=B \backslash \mathbf{Q}^{n}$. The set $E$ is finely open and has fine closure $\bar{E}^{p}=\bar{B}$. Hence $\partial_{p} E=\partial B \cup\left(B \cap \mathbf{Q}^{n}\right)$, while $\partial E=\bar{B}$.

9.1 Acknowledgments. Part of this research was done during several visits of the third author to Linköpings universitet in 2012-2014 and while all three authors visited Institut Mittag-Leffler in the autumn of 2013. We thank both institutions for their hospitality and support.

Open Access. This article is distributed under the terms of the Creative Commons Attribution License which permits any use, distribution, and reproduction in any medium, provided the original author(s) and the source are credited.

\section{REFERENCES}

[1] D. R Adams and L. I. Hedberg, Function Spaces and Potential Theory, Springer, BerlinHeidelberg, 1996.

[2] D. R. Adams and J. L. Lewis, Fine and quasiconnectedness in nonlinear potential theory, Ann. Inst. Fourier (Grenoble) 35 (1985), 57-73.

[3] L. Ambrosio, M. Colombo, and S. Di Marino, Sobolev spaces in metric measure spaces: reflexivity and lower semicontinuity of slope, Variational Methods for Evolving Objects, Math. Soc. Japan, Tokyo, 2015, pp. 1-58.

[4] L. Ambrosio, N. Gigli, and G. Savaré, Density of Lipschitz functions and equivalence of weak gradients in metric measure spaces, Rev. Mat. Iberoam. 29 (2013), 969-996.

[5] A. Björn, Characterizations of p-superharmonic functions on metric spaces, Studia Math. 169 (2005), 45-62.

[6] A. Björn, A weak Kellogg property for quasiminimizers, Comment. Math. Helv. 81 (2006), 809825.

[7] A. Björn, Removable singularities for bounded p-harmonic and quasi(super)harmonic functions on metric spaces, Ann. Acad. Sci. Fenn. Math. 31 (2006), 71-95.

[8] A. Björn and J. Björn, Boundary regularity for p-harmonic functions and solutions of the obstacle problem on metric spaces, J. Math. Soc. Japan 58 (2006), 1211-1232. 
[9] A. Björn and J. Björn, Nonlinear Potential Theory on Metric Spaces, European Math. Soc., Zürich, 2011.

[10] A. Björn and J. Björn, Obstacle and Dirichlet problems on arbitrary nonopen sets in metric spaces, and fine topology, Rev. Mat. Iberoam. 31 (2015), 161-214.

[11] A. Björn, J. Björn, and V. Latvala, The weak Cartan property for the p-fine topology on metric spaces, Indiana Univ. Math. J. 64 (2015), 915-941.

[12] A. Björn, J. Björn, and V. Latvala, Sobolev spaces, fine gradients and quasicontinuity on quasiopen sets in $\mathbf{R}^{n}$ and metric spaces, Ann. Acad. Sci. Fenn. Math. 41 (2016), 551-560.

[13] A. Björn, J. Björn, and M. Parviainen, Lebesgue points and the fundamental convergence theorem for superharmonic functions on metric spaces, Rev. Mat. Iberoam. 26 (2010), 147-174.

[14] A. Björn, J. Björn, and N. Shanmugalingam, The Dirichlet problem for p-harmonic functions on metric spaces, J. Reine Angew. Math. 556 (2003), 173-203.

[15] A. Björn, J. Björn, and N. Shanmugalingam, Quasicontinuity of Newton-Sobolev functions and density of Lipschitz functions on metric spaces, Houston J. Math. 34 (2008), 1197-1211.

[16] A. Björn and N. Marola, Moser iteration for (quasi)minimizers on metric spaces, Manuscripta Math. 121 (2006), 339-366.

[17] J. Björn, Boundary continuity for quasiminimizers on metric spaces, Illinois J. Math. 46 (2002), 383-403.

[18] J. Björn, Wiener criterion for Cheeger p-harmonic functions on metric spaces, Potential Theory in Matsue, Math. Soc. Japan, Tokyo, 2006, pp. 103-115.

[19] J. Björn, Fine continuity on metric spaces, Manuscripta Math. 125 (2008), 369-381.

[20] J. Björn, P. MacManus, and N. Shanmugalingam, Fat sets and pointwise boundary estimates for p-harmonic functions in metric spaces, J. Anal. Math. 85 (2001), 339-369.

[21] M. Brelot, Sur la théorie moderne du potentiel, C. R. Acad. Sci. Paris 209 (1939), 828-830.

[22] M. Brelot, Points irréguliers et transformations continues en théorie du potentiel J. Math. Pures Appl. (9) 19 (1940), 319-337.

[23] M. Brelot, Sur les ensembles effilés, Bull. Sci. Math. (2) 68 (1944), 12-36.

[24] M. Brelot On Topologies and Boundaries in Potential Theory, Springer, Berlin-Heidelberg, 1971.

[25] H. Cartan, Théorie générale du balayage en potentiel newtonien Ann. Univ. Grenoble Sect. Sci. Math. Phys. (N.S.) 22 (1946), 221-280.

[26] J. Cheeger, Differentiability of Lipschitz functions on metric measure spaces, Geom. Funct. Anal. 9 (1999), 428-517.

[27] G. Choquet, Sur les points d'effilement d'un ensemble. Application à l'étude de la capacité, Ann. Inst. Fourier (Grenoble) 9 (1959), 91-101.

[28] B. Franchi, P. Hajłasz and P. Koskela, Definitions of Sobolev classes on metric spaces, Ann. Inst. Fourier (Grenoble) 49 (1999), 1903-1924.

[29] B. Fuglede, The quasi topology associated with a countably subadditive set function, Ann. Inst. Fourier (Grenoble) 21 (1971), 123-169.

[30] B. Fuglede, Finely Harmonic Functions, Springer, Berlin-New York, 1972

[31] P. Hajłasz and P. Koskela, Sobolev met Poincaré, Mem. Amer. Math. Soc. 145 (2000), no. 688.

[32] L. I. Hedberg, Non-linear potentials and approximation in the mean by analytic functions, Math. Z. 129 (1972), 299-319.

[33] L. I. Hedberg and T. H. Wolff, Thin sets in nonlinear potential theory, Ann. Inst. Fourier (Grenoble) 33 (1983), 161-187.

[34] J. Heinonen, Lectures on Analysis on Metric Spaces, Springer, New York, 2001.

[35] J. Heinonen, T Kilpeläinen, and J. Malý, Connectedness in fine topologies, Ann. Acad. Sci. Fenn. Ser. A I Math. 15 (1990), 107-123. 
[36] J. Heinonen, T. Kilpeläinen, and O. Martio, Nonlinear Potential Theory of Degenerate Elliptic Equations, 2nd ed., Dover, Mineola, NY, 2006.

[37] J. Heinonen and P. Koskela, Quasiconformal maps in metric spaces with controlled geometry, Acta Math. 181 (1998), 1-61.

[38] J. Heinonen, P. Koskela, N. Shanmugalingam, and J. T. Tyson, Sobolev Spaces on Metric Measure Spaces, Cambridge Univ. Press, Cambridge, 2015.

[39] S. Keith, Measurable differentiable structures and the Poincaré inequality, Indiana Univ. Math. J. 53 (2004), 1127-1150.

[40] T. Kilpeläinen and J. Malý, The Wiener test and potential estimates for quasilinear elliptic equations, Acta Math. 172 (1994), 137-161.

[41] J. Kinnunen and V. Latvala, Fine regularity of superharmonic functions on metric spaces, Future Trends in Geometric Function Theory, Univ. Jyväskylä, Jyväskylä, 2003, pp. 157-167.

[42] J. Kinnunen and O. Martio, Nonlinear potential theory on metric spaces, Illinois J. Math. 46 (2002), 857-883.

[43] J. Kinnunen and N. Shanmugalingam, Regularity of quasi-minimizers on metric spaces, Manuscripta Math. 105 (2001), 401-423.

[44] R. Korte, A Caccioppoli estimate and fine continuity for superminimizers on metric spaces, Ann. Acad. Sci. Fenn. Math. 33 (2008), 597-604.

[45] P. Koskela and P. MacManus, Quasiconformal mappings and Sobolev spaces, Studia Math. 131 (1998), 1-17.

[46] V. Latvala, Finely superharmonic functions of degenerate elliptic equations, Ann. Acad. Sci. Fenn. Ser. A I Math. Dissertationes 96 (1994).

[47] J. Lukeš, J. Malý, and L. Zajíček, Fine Topology Methods in Real Analysis and Potential Theory, Springer, Berlin-Heidelberg, 1986

[48] J. Malý and W. P. Ziemer, Fine Regularity of Solutions of Elliptic Partial Differential Equations, Amer. Math. Soc., Providence, RI, 1997.

[49] P. Mikkonen, On the Wolff potential and quasilinear elliptic equations involving measures, Ann. Acad. Sci. Fenn. Math. Diss. 104 (1996).

[50] N. Shanmugalingam, Newtonian spaces: An extension of Sobolev spaces to metric measure spaces, Rev. Mat. Iberoam. 16 (2000), 243-279.

[51] N. Shanmugalingam, Harmonic functions on metric spaces, Illinois J. Math. 45 (2001), 10211050 .

[52] N. Wiener, The Dirichlet problem, J. Math. Phys. 3 (1924), 127-146.

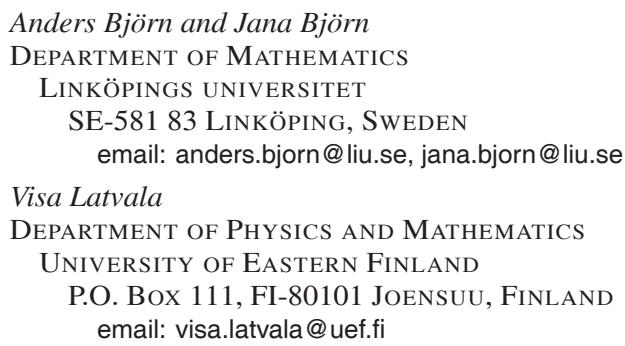

(Received October 20, 2014 and in revised form April 28, 2015) 